\title{
Gastric mucosal devitalization improves blood pressure, renin and cardiovascular lipid deposition in a rat model of obesity
}

\section{다 (1) $\odot$}

\section{Authors}

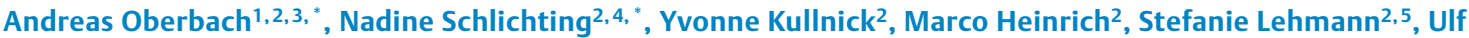

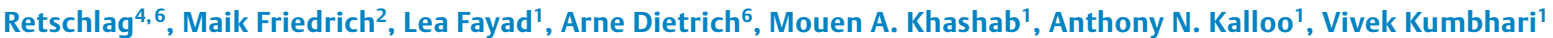

Institutions

1 Department of Medicine and Division of Gastroenterology and Hepatology. The Johns Hopkins Medical Institutions, Baltimore, Maryland, United States

2 Fraunhofer Institute for Cell Therapy and Immunology, Department of Diagnostics, Leipzig, Germany

3 Department of Cardiac Surgery, Ludwig-MaximiliansUniversity, Munich, Germany

4 Department of Visceral, Transplantation, Thoracic and Vascular Surgery, Leipzig University Hospital, Leipzig, Germany

5 Department of Internal Medicine, University of Leipzig, Leipzig, Germany

6 Integrated Research and Treatment Centre Obesity Diseases, Leipzig University, Leipzig, Germany

submitted 8.5.2019

accepted after revision 25.6.2019

Bibliography

DOI https://doi.org/10.1055/a-0990-9683 |

Endoscopy International Open 2019; 07: E1605-E1615

(c) Georg Thieme Verlag KG Stuttgart · New York

eISSN 2196-9736

\section{Corresponding author}

Vivek Kumbhari, Assistant Professor of Medicine, Division of Gastroenterology and Hepatology, Director of Bariatric Endoscopy, Johns Hopkins Medical Institutions, Sheikh Zayed Building, 1800 Orleans Street, Suite 7125G, Baltimore, MD 21287

vkumbhari@gmail.com

Supplementary material, Supplementary Table 1

Online content viewable at:

https://doi.org/10.1055/a-0990-9683

\section{ABSTRACT}

Background and study aims In lieu of the drawbacks of metabolic surgery, a method of mimicking resection of the gastric mucosa could be of value to those with obesityrelated cardiovascular disease (CVD). Our study aims to investigate the effect of gastric mucosal devitalization (GMD) on blood pressure (BP) and cardiovascular lipid deposition in a rat model of obesity.

Methods GMD of $70 \%$ of the stomach was achieved by argon plasma coagulation. GMD was compared to sleeve gastrectomy (SG) and sham (SH) in a high-fat-diet-induced rat model of obesity (48 rats). At 8 weeks, we measured noninvasive $\mathrm{BP}$, renin, vessel relaxation and ghrelin receptor regulation in the aorta. In addition, we quantified cardiac lipid deposition and lipid droplet deposition in cardiac muscle and aorta.

Results GMD and SG were observed to have similar reductions in body weight, visceral adiposity, and serum lipid profile compared to SH rats. GMD resulted in a significant reduction in arterial BP compared to SH. Furthermore, there were significant reductions in plasma renin activity and percentage of phenylnephrine constriction to acetylcholine at the aortic ring in GMD rats compared to $\mathrm{SH}$, providing insights into the mechanisms behind the reduced BP. Interestingly, the reduced BP occurred despite a reduction in endothelial ghrelin recteptor activation. Cardiac lipid content was significantly reduced in GMD rats. Lipid deposition, as illustrated by Nile Red stain, was reduced in cardiac muscle and the aorta.

Conclusion GMD resulted in a significant improvement in $\mathrm{BP}$, renin and cardiovascular lipid deposition. GMD deserves further attention as a method of treating obesity-related CVD.

\footnotetext{
* These authors contributed equally
} 


\section{Introduction}

Despite increased public awareness of the epidemic of obesity, the percentage of the population afflicted with obesity and its associated comorbidities, particularly cardiovascular disease (CVD), continues to rise [1]. Widely accepted methods of treating obese patients with CVD are diet and lifestyle therapies, pharmacotherapy and metabolic surgery [2]. Metabolic surgery, also know as bariatric surgery, is currently the most effective treatment for obesity and its associated cardiovascular comorbidities, such as hypertension, cerebrovascular disease, congestive heart failure and myocardial infarction [3,4] The benefits of metabolic surgery go beyond their ability to simply produce weight loss and these surgeries alter critical signaling and metabolic pathways [5-7]. However, as they are invasive and involve multiple irreversible alterations to the gastrointestinal tract, widespread dissemination is unlikely to occur $[8,9]$. Furthermore, metabolic surgery, due to its risk profile, is not a suitable strategy to prevent obesity-related CVD. Therefore, there arises a need to decipher the component of metabolic surgery that yields the greatest benefit to facilitate development of targeted minimally invasive therapies $[10,11]$.

We have been investigating the hypothesis that excision of the gastric mucosa is the key component to weight-independent mechanisms observed after sleeve gastrectomy (SG) [12-14]. Our previously published works have investigated an alternative to excision, devitalization of the gastric mucosa, in an obese rat and porcine model $[12,13]$. We have elucidated that gastric mucosal devitalization (GMD) in a high-fat diet induced rat model of obesity resulted in reduced body weight and visceral adiposity, improved serum lipid profile and markers of insulin resistance, and reduced liver lipid content [12]. In our porcine study, we demonstrated that GMD resulted in significant relative reductions in body weight and visceral adiposity, as well as a significant reduction in liver, skeletal and cardiac muscle lipid droplet (LD) deposition [13]. In light of these encouraging outcomes, we investigated the influence of GMD on the most sinister of obesity-related comorbidities, cardiovascular-related diseases.

Our hypothesis was that GMD would result in a reduction in blood pressure as well as reduction in LD deposition in cardiac muscle and aorta. Blood pressure was a selected outcome due to hypertension's known association with debilitating comorbidities such as heart, cerebrovascular, and renal disease [15]. Quantification of LD deposition in cardiac muscle was evaluated because cardiac lipotoxicity is a key contributor to heart failure, a sinister and often irreversible morbidity [16]. To investigate our hypothesis that the gastric mucosa is a valuable target for treatment of CVD, we used our previously validated high-fat diet (HFD) rat model and method of GMD to selectively eradicate the gastric mucosa, without altering gastric volume or intestinal anatomy [12]. To assess the independent effects of the gastric mucosa, we included a rat model of SG, which combines excision of gastric mucosa with the addition of a reduction in gastric volume.

\section{Materials and methods}

\section{Animals}

All rat procedures followed international guidelines for prevention of animal cruelty and were approved by the "Landesdirektion" Leipzig, the local authority for animal care [17]. Fourweek-old male Sprague-Dawley rats ( $n=48$; MEZ, Medical Experimental Center, University of Leipzig, Leipzig, Germany; $100-150 \mathrm{~g}$ ) were used. Further details can be found in the Supplementary Methods section.

\section{Study design}

We used our diet-induced rat model of obesity characterized by increased body weight, visceral and subcutaneous adiposity, dyslipidemia and impaired glucose metabolism [12, 18, 19]. In Part 1, we validated our HFD-induced model of obesity. Sixteen rats were randomized into two groups receiving either chow diet $(C D, n=8)$ or high-fat diet (HFD, $n=8)$ with free access to food and water. Body weight, body mass index (BMI), visceral and subcutaneous adiposity, glucose, insulin, homeostatic model assessment for insulin resistance (HOMA-IR) and inflammatory markers were measured after an additional 11 weeks. In Part 2, we compared GMD, SG, sham (SH) and CD. We used another 24 rats that had been fed a HFD for 11 weeks and randomized them into three intervention cohorts (GMD, $\mathrm{n}=8$; $\mathrm{SG}$, $\mathrm{n}=8$; $\operatorname{sham}(\mathrm{SH}), \mathrm{n}=8)$ and one $\mathrm{CD}$ cohort $(\mathrm{CD}, \mathrm{n}=8)$ to assess a variety of outcomes after an additional 8 weeks. HFD was continued in all intervention groups during the subsequent 8-week period until sacrifice (age 23 weeks).

Outcomes assessed included body composition and serum metabolic profile, serum orexigenic and anorexigenic hormones, blood pressure and plasma renin, aortic function after concentration-dependent stimulation. endothelial ghrelin receptor abundance in the aorta and abundance of lipid content and lipid droplet associated proteins in cardiac muscle and aorta. Detailed descriptions of the methods of measurement of the above outcomes can be found in the Supplementary Methods section.

\section{Interventions}

The interventions were performed in an identical manner to that reported in our original GMD study in a rat model and further details can be found in the methods and Supplementary Methods section of that manuscript [12].

\section{Gastric mucosal devitalization (GMD)}

After a laparotomy incision and mobilization of the stomach outside the abdominal cavity, a small gastric incision in the fundus was followed by insertion of a 2-mm rigid endoscope and a 1.5-mm argon plasma coagulation (APC) probe (VIO 300 D/ APC2-HF-generator; ERBE Elektromedizin, Tubingen, Germany). The activated probe was then fired (Pulsed APC, effect 2 at $25 \mathrm{~W}$ with an argon flow rate of $0.2 \mathrm{~L} / \mathrm{min}$ ) using a non-contact technique for 30 seconds. We selectively ablated $70 \%$ of the surface area of the stomach along the greater curvature aspect to match the amount of mucosa removed at SG. 


\section{Sleeve gastrectomy (SG)}

A laparotomy incision was performed and the stomach mobilized outside the abdominal cavity. The lateral $70 \%$ of the stomach was excised using a TX30B 30-mm staple gun (Johnson \& Johnson MEDICAL GmbH, Norderstedt, Germany) leaving a tubular gastric remnant in continuity with the esophagus and duodenum.

\section{Sham surgery (SH)}

A laparotomy incision was made and the stomach mobilized outside the abdominal cavity. A gastric incision was performed to allow entry of an $8 \mathrm{Fr}$ catheter and the stomach lavaged with $20 \mathrm{~mL}$ of sterile water at $37^{\circ} \mathrm{C}$.

\section{Postoperative care}

Postoperative care included daily subcutaneous injection of antibiotics $(0.1 \mathrm{~mL} / 100 \mathrm{~g}$ ceftriaxone-ratiopharm 1.0 , Ratiopharm $\mathrm{GmbH}$, Ulm, Germany) for 5 days and daily admixing of analgesic (0.5 mL Metamizol (Ratiopharm GmbH, Ulm, Germany) + 30 $\mathrm{mL} 20 \%$ glucose $+70 \mathrm{~mL}$ water to the water for 2 days). Diet was resumed ad libitum on post-intervention Day 2 .

\section{Statistical analysis}

Data analysis was performed using GraphPad Prism v6.0 (GraphPad Software, La Jolla, California, United States).

Data are shown as mean \pm SD. Significant differences between the groups are indicated by bars and/or asterisks $(P<.05)$. One asterisk signifies $P<.05$, two asterisks $P<.01$, three asterisks $P<.001$ and four asterisks $P<.0001$. Further details on statistical analysis can be found in the Supplementary Methods section.

\section{Results}

\section{Part 1, Validation of the model}

\section{HFD results in increased body weight and cardiovascular alterations}

HFD resulted in a significant increase in body weight (31\%), visceral adiposity (39\%) compared to CD after 11 weeks ( $\triangleright \mathrm{Ta}$ ble 1). Serum lipid and glucose profiles were appropriately deranged in HFD rats. There were no differences in abundance of serum gut hormones (ghrelin, GLP-1 and PYY) after 11 weeks of HFD. Noninvasive systolic $(132 \pm 3 \mathrm{mmHg}$ vs $116 \pm 4 \mathrm{mmHg}, P<$ $0.001)$ and diastolic $(99 \pm 4 \mathrm{mmHg}$ vs $92 \pm 3 \mathrm{mmHg}, P<0.01)$ blood pressure were significantly increased in HFD compared to CD ( $\vee$ Fig. 1a). Further results are found in the Supplementary Results section.

- Table 1 Characteristics of the study population phenotype.

\begin{tabular}{|c|c|c|c|c|c|c|}
\hline \multirow[t]{2}{*}{ mean $\pm S D$} & \multicolumn{3}{|c|}{ 11-weeks diet } & \multicolumn{3}{|c|}{8 weeks after intervention } \\
\hline & CD & HFD & HFD + GMD & HFD + SG & $\mathrm{HFD}+\mathrm{SH}$ & CD \\
\hline $\mathrm{n}$ & 8 & 8 & 8 & 8 & 8 & 8 \\
\hline Age (weeks) & 15 & 15 & 23 & 23 & 23 & 23 \\
\hline Body weight (g) & $424 \pm 56$ & $555 \pm 35^{*}$ & $521 \pm 62^{*}$ & $586 \pm 49$ & $616 \pm 35$ & $549 \pm 34^{*}$ \\
\hline $\mathrm{BMI}\left(\mathrm{g} / \mathrm{cm}^{2}\right)$ & $0.7 \pm 0.07$ & $0.79 \pm 0.03^{*}$ & $0.73 \pm 0.06^{*}$ & $0.75 \pm 0.06^{*}$ & $0.81 \pm 0.02$ & $0.75 \pm 0.04^{*}$ \\
\hline Visceral body fat $(\mathrm{g})$ & $6.2 \pm 2.5$ & $14.8 \pm 5.3^{*}$ & $10.8 \pm 5.5^{*}$ & $13.2 \pm 6.3^{*}$ & $26.1 \pm 8.6$ & $11.7 \pm 4.8^{*}$ \\
\hline Triglycerides (mmol/L) & $0.80 \pm 0.59$ & $1.53 \pm 0.69^{*}$ & $0.78 \pm 0.17^{*}$ & $0.68 \pm 0.2^{*}$ & $1.63 \pm 0.91$ & $0.79 \pm 0.26^{*}$ \\
\hline Total cholesterol (mmol/L) & $2.49 \pm 0.89$ & $3.75 \pm 1.06^{*}$ & $1.2 \pm 0.49^{*}$ & $1.49 \pm 0.54^{*}$ & $2.95 \pm 0.76$ & $2.72 \pm 0.47$ \\
\hline HDL cholesterol (mmol/L) & $1.24 \pm 0.39$ & $1.36 \pm 0.38$ & $0.37 \pm 0.3^{*}$ & $0.41 \pm 0.25^{*}$ & $1 \pm 0.24$ & $0.92 \pm 0.28$ \\
\hline LDL cholesterol (mmol/L) & $0.89 \pm 0.52$ & $1.69 \pm 0.70 *$ & $0.48 \pm 0.3^{*}$ & $0.77 \pm 0.36$ & $1.21 \pm 0.76$ & $1.44 \pm 0.22$ \\
\hline Fasting glucose (mmol/L) & $4.9 \pm 0.6$ & $5.9 \pm 0.4^{*}$ & $5.3 \pm 0.4^{*}$ & $5.7 \pm 0.6^{*}$ & $6.4 \pm 0.5$ & $5.0 \pm 0.3^{*}$ \\
\hline Fasting insulin (mmol/L) & $1.35 \pm 0.5$ & $2.19 \pm 0.8^{*}$ & $0.93 \pm 0.6^{*}$ & $0.75 \pm 0.2^{*}$ & $1.99 \pm 0.7$ & $1.03 \pm 0.2^{*}$ \\
\hline HOMA-IR & $1.42 \pm 0.6$ & $2.77 \pm 1.1^{*}$ & $1.06 \pm 0.6^{*}$ & $0.93 \pm 0.3^{*}$ & $2.69 \pm 0.9$ & $1.10 \pm 0.2^{*}$ \\
\hline $\mathrm{CRP}(\mathrm{mg} / \mathrm{mL})$ & $0.55 \pm 0.2$ & $0.40 \pm 0.1$ & $0.66 \pm 0.1$ & $0.87 \pm 0.7$ & $0.75 \pm 0.5$ & $1.22 \pm 0.7$ \\
\hline IL-6 (pg/mL) & $243 \pm 26$ & $231 \pm 33$ & $293 \pm 81$ & $292 \pm 100$ & $255 \pm 62$ & $217 \pm 62$ \\
\hline \multicolumn{7}{|c|}{$\begin{array}{l}\text { Measurements in the animal groups at } 15 \text { weeks of age ( } 11 \text { weeks of HFD) and } 23 \text { weeks of age ( } 8 \text { weeks after intervention). Values are means + SD of dietary groups } \\
(C D \text {, chow diet; HFD, high-fat diet) and intervention groups (GMD, gastric mucosa devitalization; SG, sleeve gastrectomy; SH, sham surgery). Significant differences } \\
(P<.05) \text { between GMD and SG compared with SH are indicated by asterisks. CD, chow diet; HFD, high-fat diet; GMD, gastric mucosal devitalization; SG, sleeve gas- } \\
\text { trectomy; SH, sham operation; BMI, body mass index; LDL, low-density lipoprotein; HDL, high-density lipoprotein; HOMA-IR, homeostatic model assessment of in- } \\
\text { sulin resistance; AUC } 2 \text { h oGT, area under the curve based on five timepoints (baseline, } 15 \text { min, } 30 \text { min, } 60 \text { min, } 120 \text { min) of } 2 \text {-hour oral glucose tolerance test; CRP, } \\
\text { C-reactive protein; IL- } 6 \text {, interleukin } 6 .\end{array}$} \\
\hline
\end{tabular}



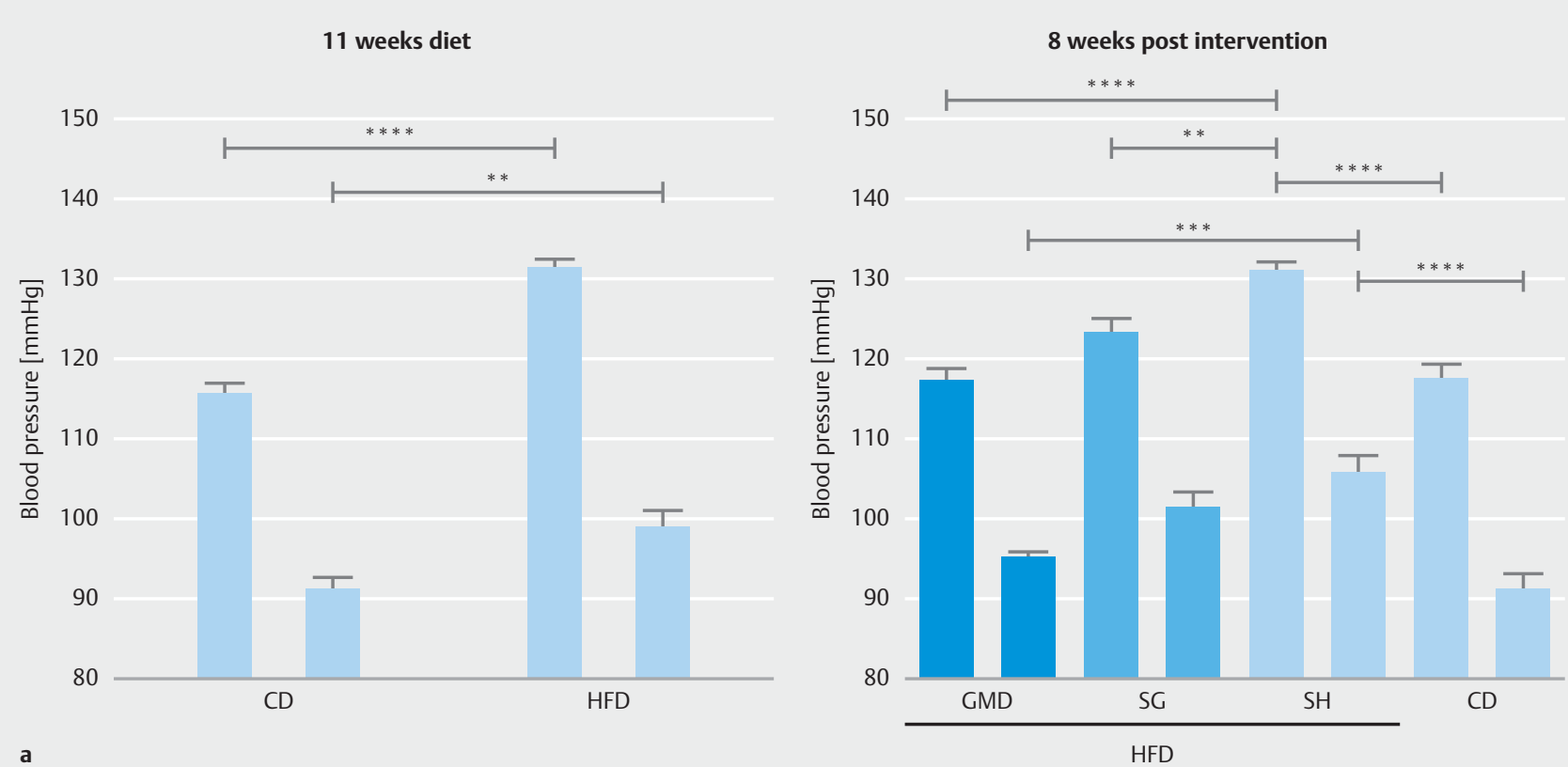

a

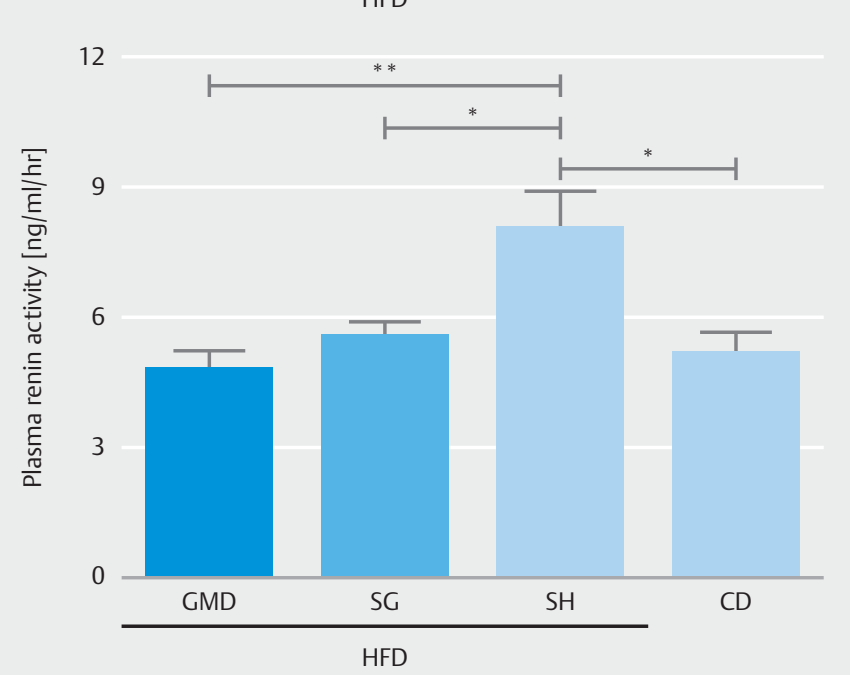

b

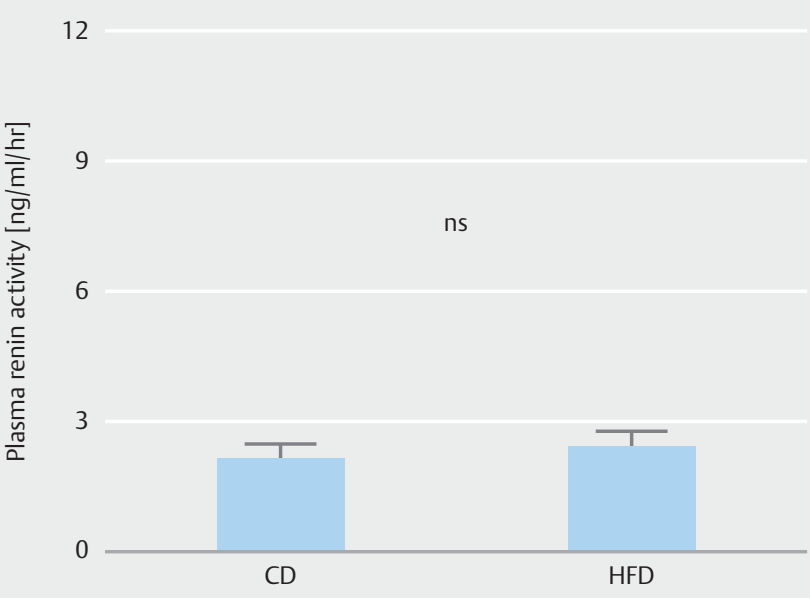

- Fig. 1 Regulation of blood pressure and renin activity in the study population. Measurements in the animal groups at 15 weeks of age (11 weeks of HFD) and 23 weeks of age (8 weeks after intervention). a Noninvasive measurement of blood pressure using a tail cuff setup revealed an increased systolic and diastolic blood pressure in rats after 11 weeks of feeding HFD compared to CD rats. Eight weeks after intervention, GMD treated rats showed significant reduction of both pressure values compared to SH. b Plasma renin activity was not altered after receiving 11 weeks HFD. Eight weeks after intervention, renin activity was significantly reduced in GMD and SG rats. CD, chow diet; HFD, high-fat diet; GMD, gastric mucosal devitalization; SG, sleeve gastrectomy; SH, sham operation

\section{Part 2 - Randomized comparison effects of GMD, SG and $\mathrm{SH}$ in HFD rats}

Results of our technique of using APC to selectively devitalize the gastric mucosa, without damage to deeper tissue layers or alteration in gastric volume have been previously reported [12].

\section{Body composition and serum metabolic profile}

GMD resulted in a significant reduction in body weight $(521 \pm$ $62 \mathrm{~g}$ vs $616 \pm 35 \mathrm{~g}, P<0.01)$ and visceral adiposity $(10.8 \pm 5.5 \mathrm{~g}$ vs $26.1 \pm 8.6 \mathrm{~g}, P<0.001$ ) compared to $\mathrm{SH}$ at 8 weeks ( $\triangleright$ Table 1). There was a significant reduction in triglycerides and total cholesterol in both GMD and SG compared to SH rats ( $>$ Table
1). GMD was able to significantly lower $L D L$ cholesterol at 8 weeks compared to $\mathrm{SH}(0.48 \pm 0.3 \mathrm{mmol} / \mathrm{L}$ vs $1.21 \pm 0.76 \mathrm{mmol} /$ $\mathrm{L}, P<0.05)$. Fasting glucose and fasting insulin were significantly lower in GMD $(5.3 \pm 0.4 \mathrm{mmol} / \mathrm{L}$ vs $6.4 \pm 0.5 \mathrm{mmol} / \mathrm{L}, P<0.001$; $0.93 \pm 0.6 \mathrm{mmol} / \mathrm{L}$ vs $1.99 \pm 0.7 \mathrm{mmol} / \mathrm{L}, P<0.001)$ and SG $(5.7 \pm$ $0.6 \mathrm{mmol} / \mathrm{L}$ vs $6.4 \pm 0.5 \mathrm{mmol} / \mathrm{L}, P<0.05 ; 0.75 \pm 0.2 \mathrm{mmol} / \mathrm{L}$ vs $1.99 \pm 0.7 \mathrm{mmol} / \mathrm{L}, P<0.01)$ rats compared to $\mathrm{SH}$. To illustrate changes in insulin resistance, HOMA-IR was also significantly lower in GMD $(1.06 \pm 0.6$ vs $2.69 \pm 0.9, P<0.001)$ and SG $(0.93 \pm$ 0.3 vs $2.69 \pm 0.9, P<0.001)$ rats compared to $\mathrm{SH}$. There were no significant differences with regards to $C$-reactive protein (CRP) and Interleukin-6 (IL-6) ( Table 1). 


\section{Regulation of serum orexigenic and anorexigenic hormones}

There was a high biological variation in abundance of serum ghrelin, GLP-1 and PYY when measured after being fasted for 10 hours or following oral glucose stimulation by a 2-hour oral glucose tolerance test (OGTT) (Supplementary Table 1). Fasting total serum ghrelin levels were significantly reduced in GMD compared to $\mathrm{SH}$ at 8 weeks $(0.81 \pm 0.29 \mathrm{ng} / \mathrm{mL}$ vs $1.43 \pm$ $0.58 \mathrm{ng} / \mathrm{mL}, P<0.05)$. However, fasting active ghrelin levels were not significantly different between GMD, SG and SH (Supplementary Table 1). The 2-hour OGTT (presented as area under the curve $[A U C]$ ) in the GMD group did not reveal a significant reduction in total ghrelin, but surprisingly, did show a significant increase in active AUC active ghrelin compared to $\mathrm{SH}$ (132 \pm 45 vs $81 \pm 25, P<0.05)$ (Supplementary Table 1 ).

With regards to the other measured gut hormones, fasting total GLP-1 was significantly higher in GMD compared to SH (29.2 $\pm 6.3 \mathrm{pmol} / \mathrm{L}$ vs $14.9 \pm 7.2 \mathrm{pmol} / \mathrm{L}, P<0.05)$ (Supplementary Table 1). The 2-hour OGTT revealed a significant increase in total GLP-1 in SG compared to SH rats $(29 \pm 17 \mathrm{pmol} / \mathrm{L}$ vs $14.9 \pm 7.2 \mathrm{pmol} / \mathrm{L}, P<0.05)$. Such statistical significance was not reached in the GMD cohort. Neither fasting nor 2-hour OGTT Neuropeptide PYY was not significantly different between the intervention cohorts (Supplementary Table 1).

\section{Blood pressure and plasma renin}

Systolic and diastolic blood pressure were significantly decreased in GMD rats compared to $\mathrm{SH}(117 \pm 5 \mathrm{mmHg}$ vs $131 \pm$ $3 \mathrm{mmHg}, P<0.001)$ and $(95 \pm 3 \mathrm{mmHg}$ vs $106 \pm 6 \mathrm{mmHg}, P<$ 0.001 ), respectively ( $>$ Fig. 1 a). Systolic blood pressure was significantly reduced in SG compared to $\mathrm{SH}$ rats $(124 \pm 4 \mathrm{mmHg}$ vs $131 \pm 6 \mathrm{mmHg}, P<0.01)$ with reductions in diastolic blood pressure not meeting statistical significance ( $\mathbf{F i g . 1 a )}$ ). Plasma renin activity was significantly lower in the GMD cohort compared with $\mathrm{SH}(4.75 \pm 1.16 \mathrm{ng} / \mathrm{mL} / \mathrm{hr}$ vs $8.24 \pm 2.63 \mathrm{ng} / \mathrm{mL} / \mathrm{hr}, P<$ $0.01)$. A similar reduction in SG rats was also observed $(5.57 \pm$ $1.27 \mathrm{ng} / \mathrm{mL} / \mathrm{hr}$ vs $8.24 \pm 2.63 \mathrm{ng} / \mathrm{mL} / \mathrm{hr}, P<0.05)$.

\section{Aortic function after concentration dependent stimulation}

The aortic rings were previously unspecifically stimulated with $80 \mathrm{nM} \mathrm{KCl}$ to visualize the maximal contraction. The ratio of $\mathrm{KCl}$ /baseline during initial contraction with $\mathrm{KCl}$ is presented in - Fig. 2a. There were no differences observed between the intervention groups. The phenylephrine (PE)-dependent contraction is plotted relative to the baseline of each group. There was a significant difference in the maximal contractile response of the thoracic aorta to PE between the intervention groups with the response being significantly higher in the GMD and SG groups compared to $\mathrm{SH}(1.54 \pm 0.14$ vs $1.22 \pm 0.15, P<0.01)$ and $(1.54 \pm 0.16$ vs $1.22 \pm 0.15, P<0.001)$, respectively ( $\vee$ Fig. 2b). The acetylcholine (ACh)-dependent relaxation was plotted relative to the respective maximal PE contraction (100nM). Vasodilation in response to $\mathrm{ACh}$ illustrated a left shift with respect to the GMD and SG cohorts compared with SH, indicating an increased sensitivity to vasodilatory stimulus ( $\bullet \mathbf{F i g . 2 c}$ ). The in- serts are calculated AUC and represent the change in contraction depending on concentration of the relative stimulus from Ach ( $\triangleright$ Fig. 2c). There was a significant reduction in AUC with regard to the vasodilation response to Ach in the GMD and SG groups compared to $\mathrm{SH},(481 \pm 81$ vs $601 \pm 65, P<0.05)$ and ( $480 \pm 107$ vs $601 \pm 65, P<0.05)$, respectively. There was no difference between the GMD and SG groups.

\section{Endothelial ghrelin receptor in rat aorta}

Ghrelin has been shown to have a depressant effect on vascular reactivity, thus promoting a vasodilatory response and subsequent reduction in BP in the rat aorta. Therefore, in view of the anticipated reduction in circulating ghrelin in the GMD and SG rats compared to $\mathrm{SH}$, we sought to investigate the ghrelin receptor response to the various interventions. For reference, we illustrate histopathological characteristics of the rat aorta in - Fig. 3a. The endothelial layer (no. 2) is where the GHSR receptor is located. Fluorescence microscopy of the aortic wall is seen in Fig. $\mathbf{3 b}$. Grossly, there is the appearance that GMD and SG have a decreased abundance of GHSR in the endothelium compared to SH. Western Blot confirmed there was a significantly reduced expression of GHSR in GMD and SG rats compared to SH ( $\triangleright$ Fig. 3c). Quantitatively, the GHSR/ G3DPH for GMD was significantly lower than $\mathrm{SH}(0.21 \pm 0.12$ [AU] vs 7.32 $\pm 5.69[\mathrm{AU}][P<0.01])$. Similar findings were observed when SG was compared to SH $(0.27 \pm 0.09$ [AU]vs $7.32 \pm 5.69[\mathrm{AU}][P<$ 0.05]) ( Fig. $3 \mathrm{~d})$.

\section{Regulation of lipid content and lipid droplet associated proteins in cardiovascular tissue}

Measures of cardiac LD content were attained through several mechanisms. Cardiac LD content was significantly reduced in the GMD cohort compared to SH $(10.9 \pm 5.2 \mu \mathrm{g} / \mathrm{mg}$ vs $26.1 \pm$ $7.6 \mu \mathrm{g} / \mathrm{mg}, \quad P<0.001$ ) ( Fig.4a). Similar findings are seen when SG is compared to SH $(13.9 \pm 8.6 \mu \mathrm{g} / \mathrm{mg}$ vs $26.1 \pm 7.6 \mu \mathrm{g} /$ $\mathrm{mg}, P<0.01)$. As a frame of reference, we illustrate H\&E of cardiac muscle in $>$ Fig. $\mathbf{4 b}$. Immunofluorescence was performed to grossly illustrate the abundance of LD content and size (Nile Red stain) in cardiac tissue as well as expression of LD associated proteins PLIN 1 and PLIN 2 after the respective interventions ( $\mathbf{F i g . 4 c}$ ). GMD resulted in a reduction in LD concentration and size compared to SH. Additionally, PLIN 1 appeared reduced in cardiac muscle in GMD rats compared to $\mathrm{SH}$, however, PLIN 2 was increased in the GMD cohort. In > Fig. 4 d, PLIN 1 and 2 were quantified by Western Blot. GMD resulted in a significant reduction in PLIN 1 compared to SH $(2.29 \pm 2.34$ [AU] vs $10.23 \pm 4.61[\mathrm{AU}], P<0.001$ ) ( $>$ Fig.4e). With respect to PLIN 2, GMD resulted in a significant increase compared to $\mathrm{SH}$ $(28.27 \pm 8.02[\mathrm{AU}]$ vs $7.09 \pm 4.13[\mathrm{AU}], P<0.001)$. Similar findings were observed in the SG cohort ( $\vee$ Fig. $4 \mathbf{e}$ ).

Immunofluorescence with Nile Red stain of cardiac muscle and aorta is illustrated in - Fig. 5. The LD content appears reduced in the GMD cohort compared to SH with similar changes observed in the SG cohort ( $\triangleright$ Fig.5a). Aortic LD content was also significantly reduced in the GMD cohort compared to the SH with similar changes observed in the SG cohort ( $\triangleright$ Fig. 5b). 


\section{1 weeks diet}

3

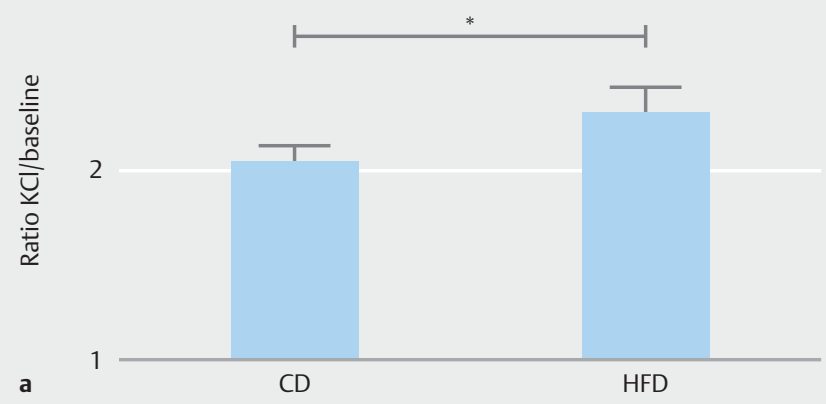

2.2

2.0

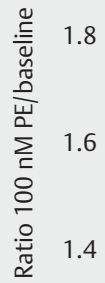

b

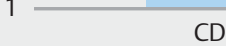

ns

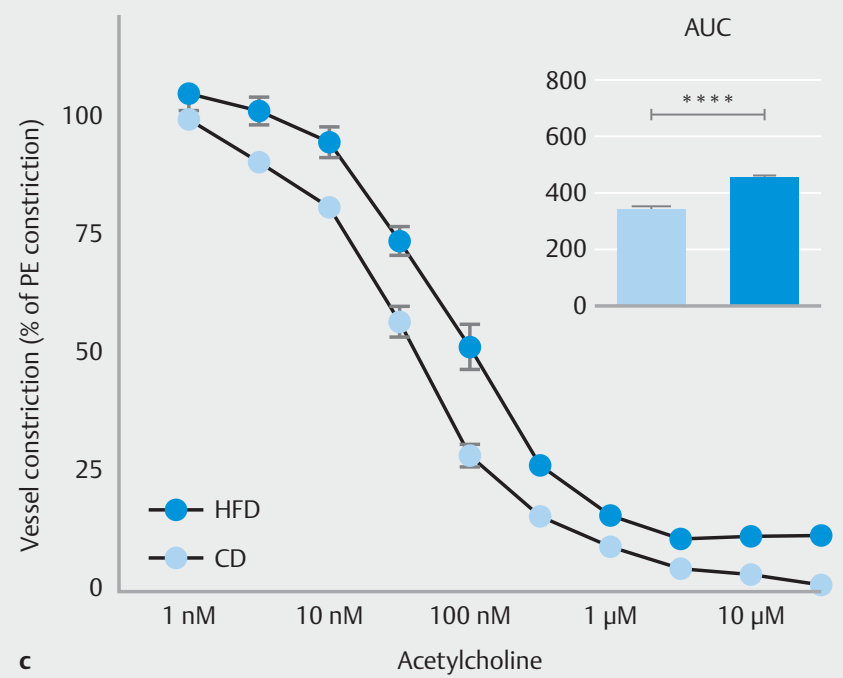

8 weeks post intervention

3

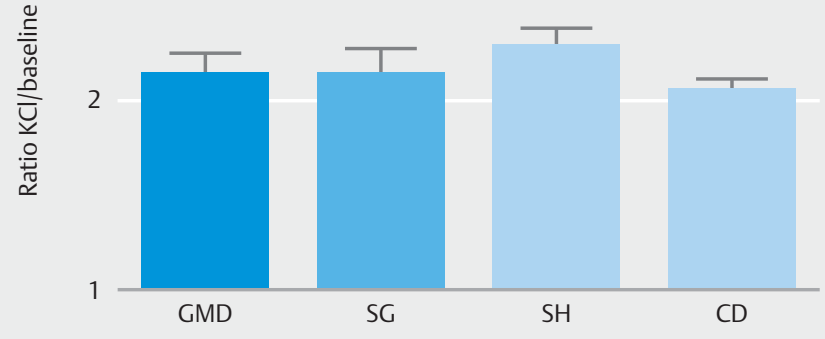

2.2

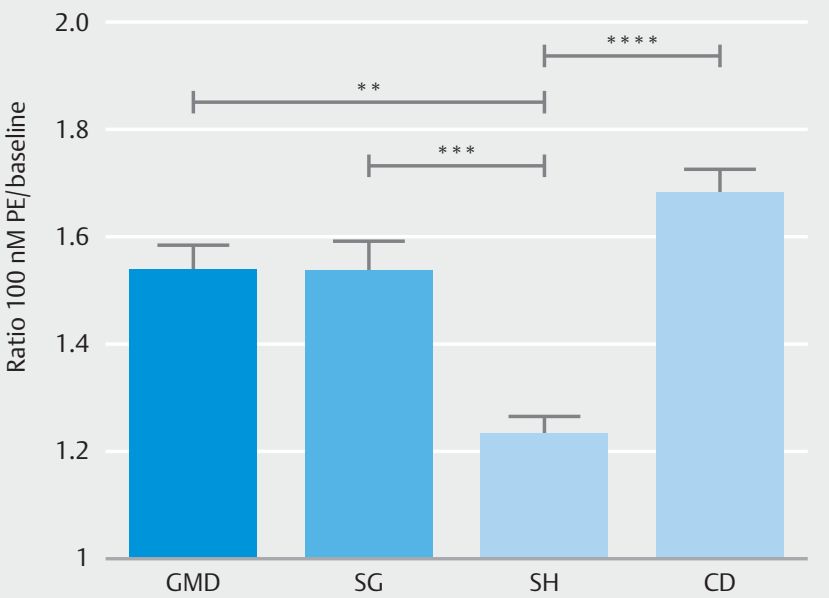

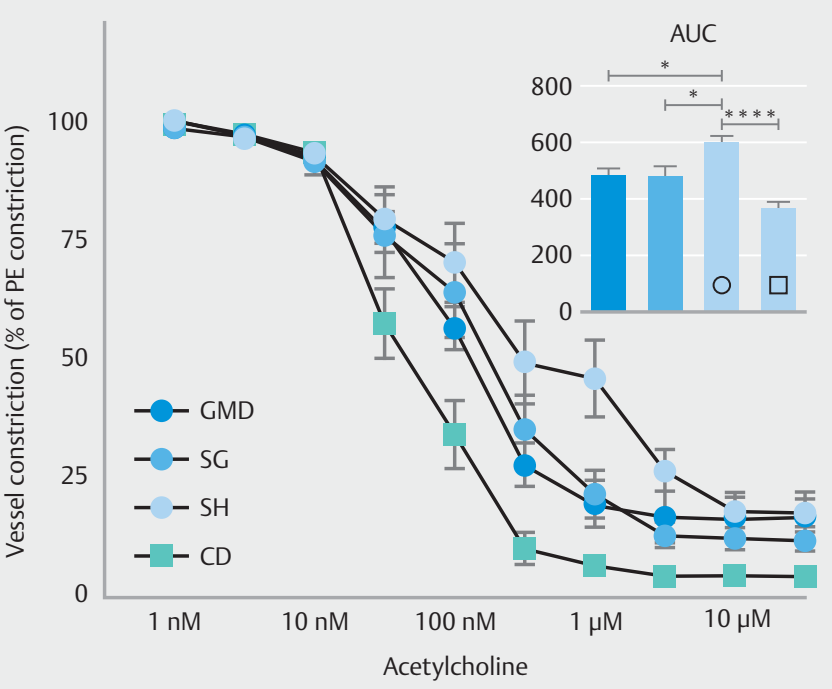

Fig. 2 Ex vivo study of response of the aorta in rats after concentration-dependent stimulation. Measurements in the animal groups at 15 weeks of age ( 11 weeks of HFD) and 23 weeks of age ( 8 weeks after intervention). a Aortic rings were stimulated with $80 \mathrm{mM}$ KCl to visualize their maximal contraction (mean \pm SEM). $\mathbf{b}$ Phenylepinephrine (PE)-dependent contraction is plotted relative to the baseline of each group. The stimulation was finished when contraction reaches $>70 \%$ of $\mathrm{KCl}$-contraction. c Acetylcholine (Ach)-dependent relaxation is plotted relative to the respective maximal PE-contraction ( $100 \mathrm{nM}$ ). Insets are calculated area under the curve (AUC; mean \pm SEM) and represent the change in contraction depending on the concentration of the relative stimulus Ach. Statistical tests used were the $t$-test and one-way ANOVA with a posthoc Tukey's - Kramer test and $P<0.05$.

$\mathrm{CD}$, chow diet; HFD, high-fat diet; GMD, gastric mucosal devitalization; SG, sleeve gastrectomy; SH, sham operation 


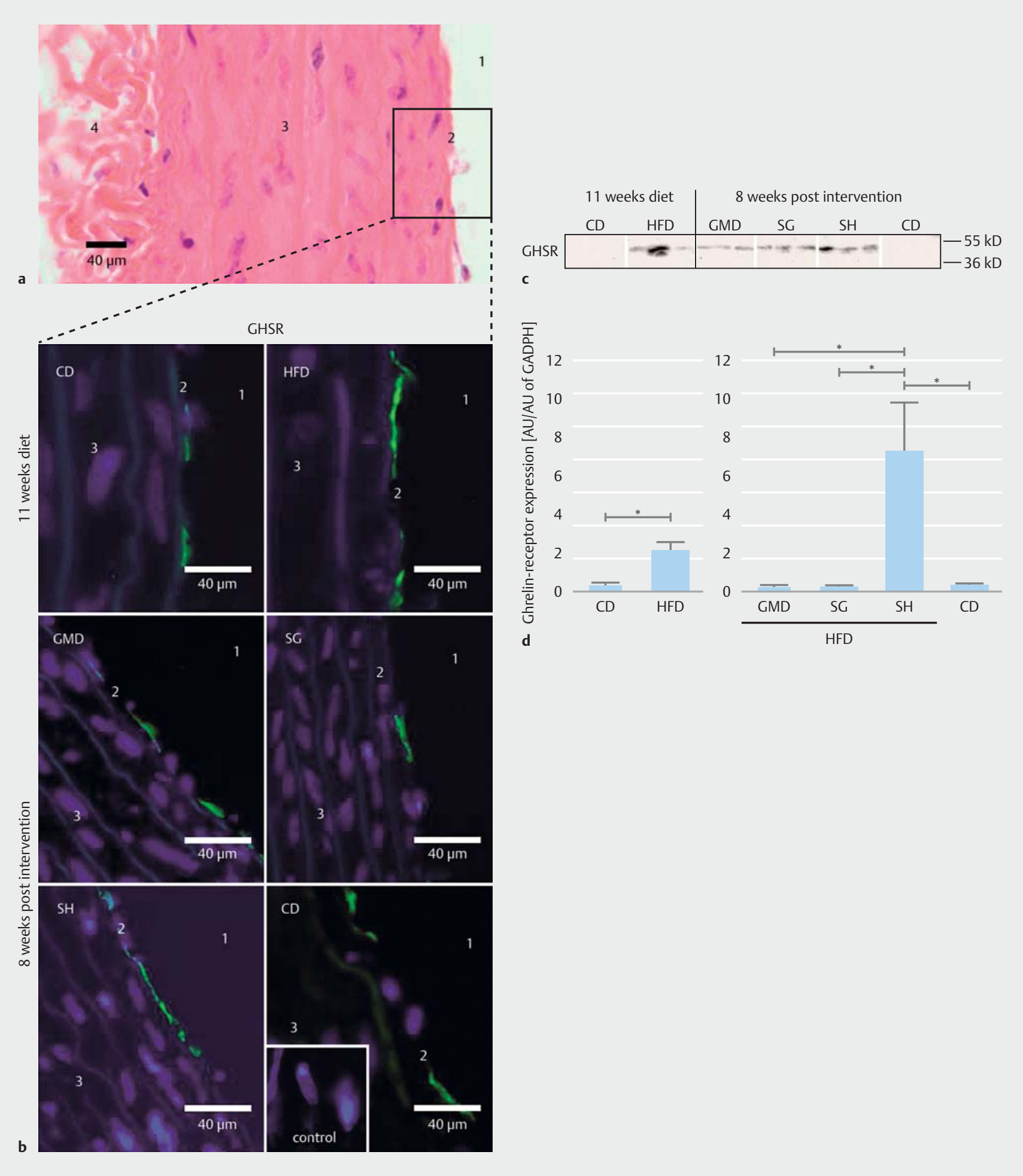

- Fig. 3 Endothelial ghrelin receptor expression in rat aorta. a HE-stain illustrates the structural characteristics of the rat aorta: 1 - Blood vessel lumen, 2 -Endothelium, 3 -Aortic smooth muscle cells, 4-Visceral fat tissue, blue-cell nuclei. b Fluorescence images of paraffin slices show Ghrelin-receptor localization (GHSR) (green) on aortic endothelium of each respective group. Nuclei - Dapi (blue), inset represents stain control without primary antibody. (C+D) Quantitative analysis of GHSR using Western Blot. GSHR, growth hormone secretagogue receptor 1a; CD, chow diet; HFD, high-fat diet; GMD, gastric mucosal devitalization; SG, sleeve gastrectomy; SH, sham operation; GAPDH, glyceraldehyde 3phosphate dehydrogenase. 


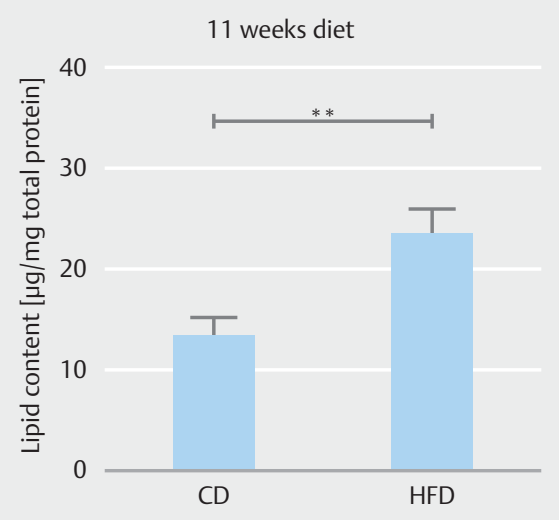

a

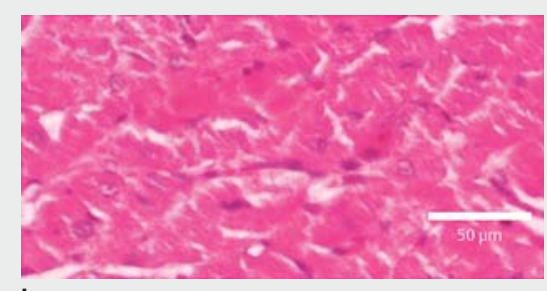

b

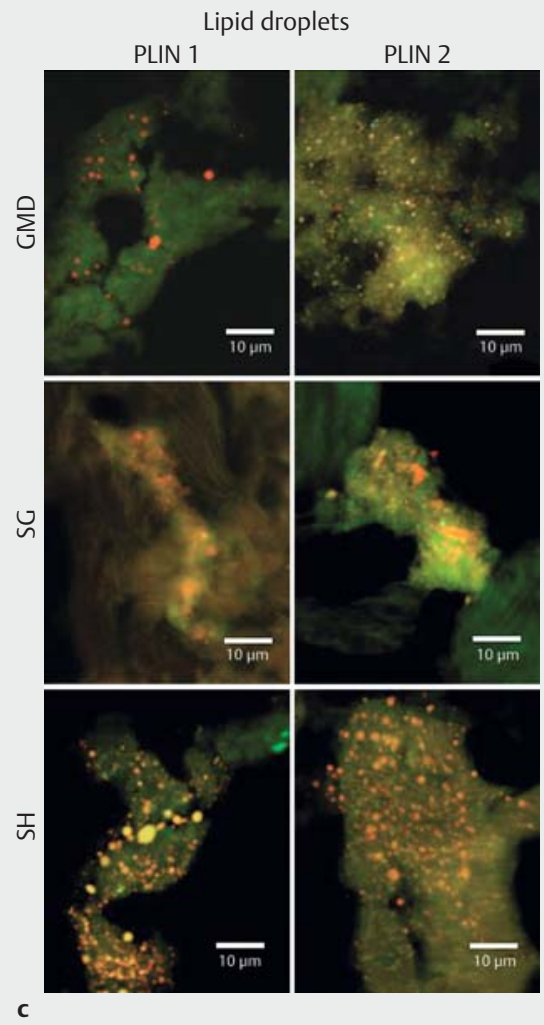

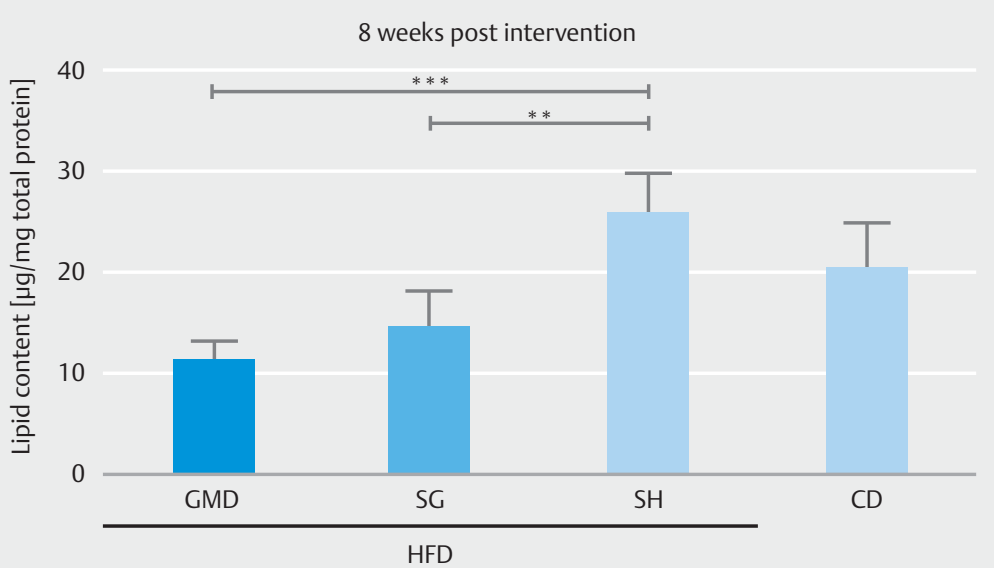

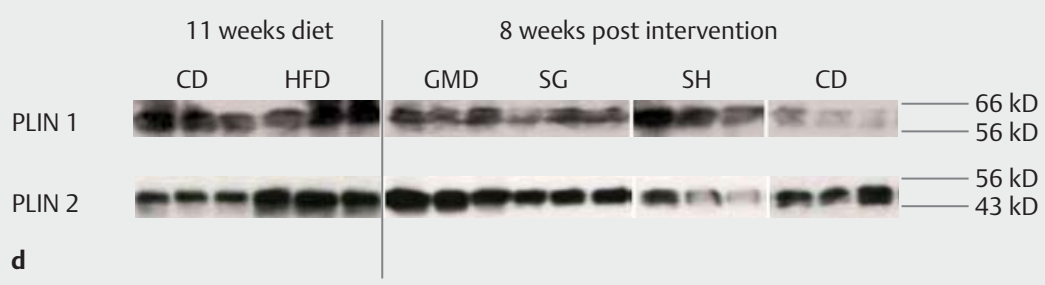

11 weeks diet

8 weeks post intervention
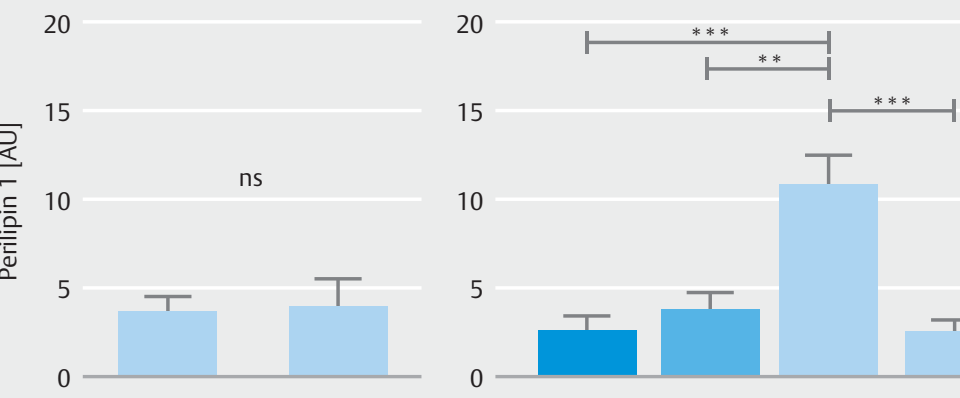

15

10

10
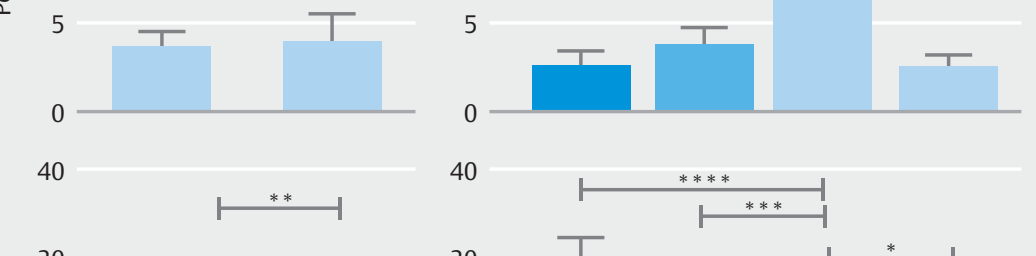

40
30

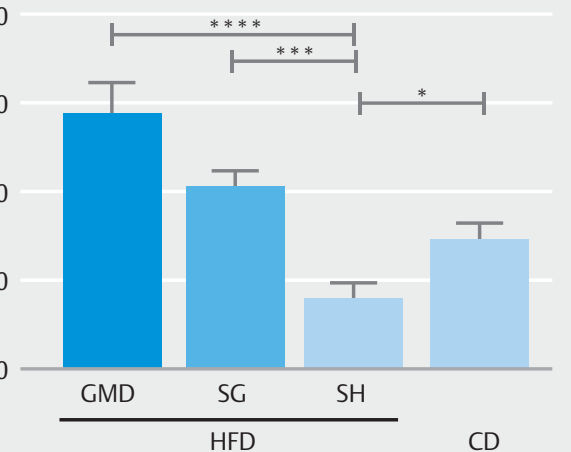

- Fig. 4 Regulation of lipid content and lipid droplet-associated proteins perilipin 1 and perilipin 2 in cardiac muscle. Measurements in the animal groups at 15 weeks of age ( 11 weeks of HFD) and 23 weeks of age ( 8 weeks after intervention). Values are means + SD of dietary groups (CD, chow diet; HFD, high-fat diet) and intervention groups (GMD, gastric mucosa devitalization; SG, sleeve gastrectomy; SH, sham surgery). Significant differences $(P<.05)$ between GMD and SG compared with SH are indicated by asterisks. a Cardiac lipid content in the respective intervention groups. Note, GMD rats had significantly reduced cardiac lipid content at 8 weeks compared to SH. b HE-stain illustrates the structural characteristics of cardiac muscle. c Immunofluorescence of cryo-slices illustrate lipid droplet concentration and size (Nile red stain) as well as expression of lipid droplet associated proteins PLIN1 and PLIN2 after 8 weeks of intervention (green). d,e Lipid droplet associated proteins were quantified by Western Blot. CD, chow diet; HFD, high-fat diet; GMD, gastric mucosal devitalization; SG, sleeve gastrectomy; SH, sham operation; PLIN1, perilipin 1; PLIN2 - perilipin 2. 

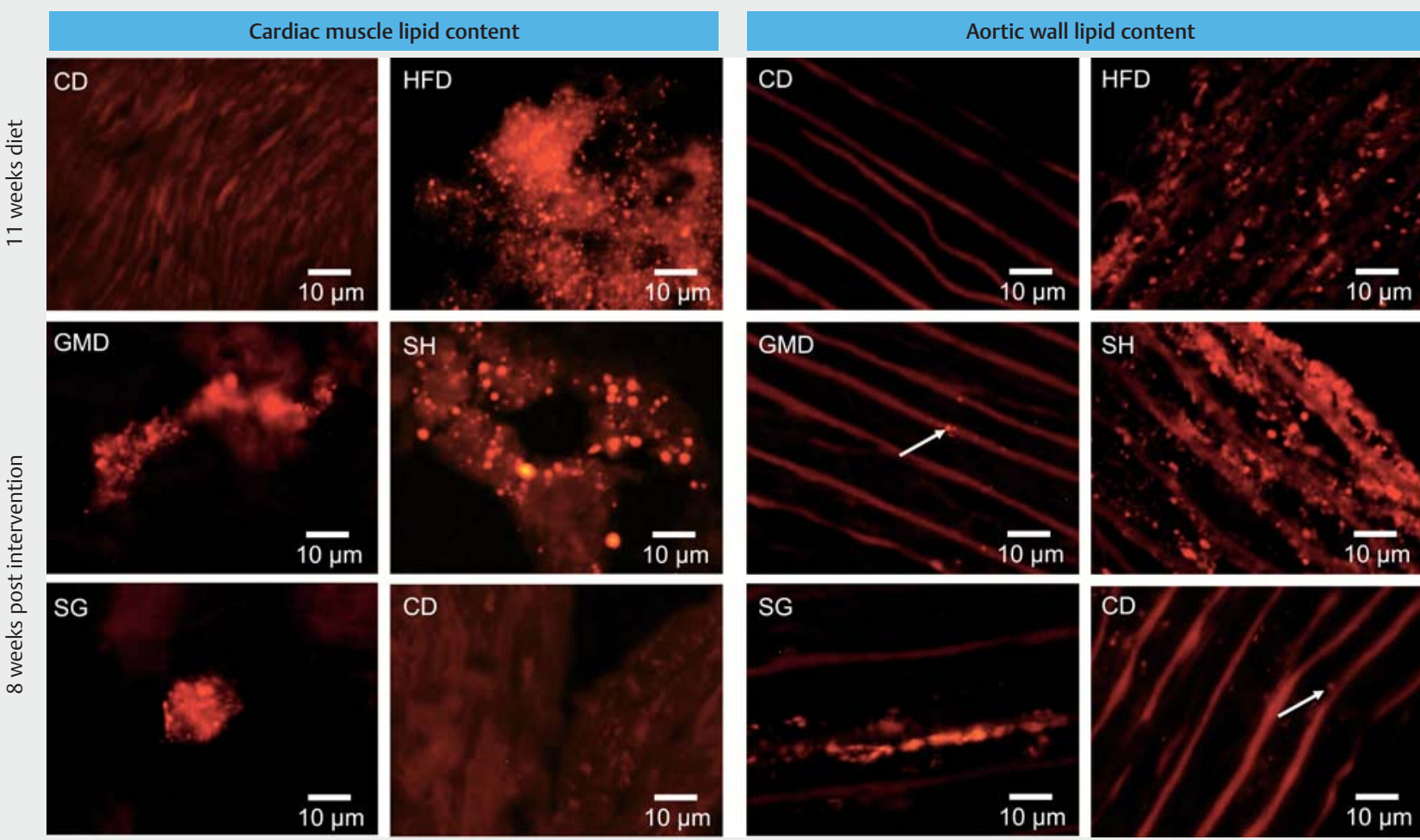

- Fig. 5 Nile Red stain of cardiac muscle and aorta. a Fluorescence staining with Nile Red illustrating cardiac muscle lipid content. b Fluorescence staining with Nile Red illustrating aortic wall lipid content. CD, chow diet; HFD, high-fat diet; GMD, gastric mucosal devitalization; SG, sleeve gastrectomy; SH, sham operation.

\section{Discussion}

Our previous work demonstrated that devitalization of the gastric mucosa, independent of reduction in gastric volume, reduced adiposity and improved lipid and glucose metabolism $[12,13]$. As a result of the observed improvement in the aforementioned metabolic effects, we hypothesized that GMD would also result in a reduction in important cardiovascular parameters such a blood pressure and cardiovascular LD deposition. In a diet-induced HFD rat model of obesity, we present novel data that GMD was indeed associated with significant improvements in blood pressure, plasma renin, as well as cardiac and aortic LD deposition. Therefore, devitalization (or potentially any other method to eliminate or exclude) of the gastric mucosa deserves further attention as a method to treat obesity associated CVD.

In NHANES 1999-2010, 35.5\% of obese individuals had hypertension and a direct causative relationship exists between overweight/obesity and hypertension [20-24]. GMD significantly reduced both systolic and diastolic BP ( $\triangleright$ Fig. 1a). This is notable as high BP is the leading cause of death worldwide [25]. Furthermore, a strong direct relationship exists between the level of BP and risk of comorbidities and death [26, 27]. Fortunately, becoming normal weight has been shown to reduce risk of developing hypertension to a level similar to those who have never been obese and a linear association exists between reduction in systolic BP and risk of mortality from CVD [2830].

The possible mechanisms behind reduction in BP observed in GMD are likely explained by the combination of hormonal effects as well as effects on vascular endothelium ( $\bullet$ Fig. 1, \ Fig. 2, > Fig. 3). Plasma renin activity was significantly lower in GMD than $\mathrm{SH}$ rats ( $\bullet \mathbf{F i g} . \mathbf{1}$ ), indicating its influence in the observed relative reduction in $\mathrm{BP}$ through its effects on the renin-angiotensin system, a major regulatory system of cardiovascular function. Furthermore, a reduction in renin has cardiovascular effects that are greater than expected by its ability to lower blood pressure alone [31,32]. A lowering of renin activity would be expected to exert potent antiatherosclerotic effects, delay the onset of type 2 diabetes and reduce frequency of atrial fibrillation and stroke $[31,33]$. The mechanism being the reduction in renin observed in GMD rats was not ascertained, however, it is known that even modest weight loss (5\%) can result in a $47 \%$ reduction in plasma renin [34].

Vascular endothelium plays an essential role in regulation of vascular tone and hence BP. Activation of muscarinic receptors in the endothelium by Ach increases the production of nitric oxide, leading to vessel relaxation. Our study showed GMD resulted in an increase in endothelium-dependent vasodilation in response to Ach compared to SH ( $\triangleright$ Fig. 2), likely contributing to lowering of BP. 
High expression of GHSR in the heart, kidney and blood vessels is evidence of ghrelin involvement in BP regulation [35]. In detail, circulating ghrelin levels inversely correlate with blood pressure, in part, due to its direct vasodilatory activities through nitric oxide and nitric oxide-independent mechanisms [35]. Therefore, in light of the finding that fasting serum ghrelin was significantly reduced in GMD rats compared to SH (Supplementary Table 1), we sought to assess the impact on blood pressure. Surprisingly, the abundance of GHSR on the endothelium of the rat aorta was significantly lower in GMD rats compared to SH ( $\mathbf{F i g . 3}$ ). This may have been expected to have a negative impact on BP, but was not observed. Such dissociative findings have been observed in studies evaluating ghrelin and GHSR, a testament to their complex interplay [35].

Obesity-associated perturbations in cardiac muscle and systemic lipid metabolism are important contributors to cardiovascular complications of obesity [16]. In concordance with the reduction in visceral adiposity, serum lipid profile and liver LD deposition seen in our earlier work, we further demonstrate a significant reduction in cardiac muscle LD deposition in GMD rats compared to SH ( $\mathbf{F i g . 4 , ~}>$ Fig.5). Accumulation of lipid in non-adipose cells of the cardiovascular system results in cellular dysfunction and death, otherwise known as lipotoxicity [16]. Increased LD deposition results in endoplasmic reticulum stress, alterations in autophagy, de novo ceremide synthesis, oxidative stress, inflammation and changes in gene expression [16]. These alterations in myocardiac structure and function directly impair cardiac function, resulting in heart failure [36]. In addition to a reduction in cardiac muscle LD, we illustrate grossly a reduction in quantity and size of LD in the aorta. Therefore, the significant reductions in cardiac muscle and aortic LD deposition have the potential to translate to clinically meaningful improvements in patients with obesity-associated CVD.

PLIN1 and PLIN2 are located on LD particle surfaces, hence, they are they are considered marker proteins for LDs [38]. They are both present in overabundance within the heart in patients with coronary artery disease [39]. GMD resulted in a reduction in PLIN1 but an increase in PLIN2 compared to SH ( $\mathbf{F i g . 4 )}$ ). The reduction in PLIN1 would imply a potential clinical benefit. The increase in PLIN2 is more difficult to interpret. Data exist demonstrating that PLIN2 is elevated in patients with CVD and atherosclerosis [40]. On the contrary, PLIN2 has been shown to improve insulin sensitivity in skeletal muscle, though this finding has not been demonstrated in cardiac muscle [41]. Further investigation into the interpretation of perilipins is warranted.

Animal models of disease have advantages as well as suffer inherent limitations. As opposed to humans, the inclusion of appropriate control groups such as sham surgery, as well as the ability to control for certain factors (environment, diet) are clear benefits. In addition, the rat model has widely available assays and antibodies allowing for detailed serum and tissue evaluation. However, the relatively young age of the rats does not allow for assessment of reversibility of chronic disease states, limiting translatability to humans. From an outcomes perspective, calculation of cardiac function was not performed, a find- ing that may have allowed demonstration of the effects of lipotoxicity on cardiac function.

\section{Conclusion}

Herein, we demonstrate that a method of selective eradication of the gastric mucosa, without alteration in the volume of the stomach, results in a significant reduction in blood pressure, plasma renin activity, and cardiac as well as aortic lipid droplet deposition. This builds on our previous work that GMD results in a reduction in visceral adiposity as well as an improvement in lipid and glucose metabolism. The collective metabolic and cardiovascular benefits observed in this high-fat-diet-induced obese rat model deserves investigation in humans. Our previous work in a large animal model demonstrating technical feasibility, efficacy and safety of GMD, provides evidence that it is time to translate GMD to a clinical setting (NCT03526263).

\section{Acknowledgements}

This study was supported by the Integrated Research and Treatment Center (IFB) Adiposity Diseases, University of Leipzig, Leipzig, Germany (sponsored by the German Federal Ministry of Education and Research) and by Erbe Elektromedizin $\mathrm{GmbH}$.

\section{Competing interests}

Dr. Kumbhari is a consultant for Apollo Endosurgery, Medtronic, ReShape Lifesciences, Boston Scientific and Pentax. He has also received research support from Apollo Endosurgery and ERBE. Mouen A. Khashab is on the medical advisory board for Boston Scientific and Olympus America and is a consultant for Boston Scientific, Olympus America, and Medtronic. Anthony N Kalloo is a founding member, equity holder, and consultant for Apollo Endosurgery.

\section{References}

[1] Ogden CL, Fakhouri TH, Carroll MD et al. Prevalence of Obesity Among Adults, by Household Income and Education - United States, 2011-2014. MMWR Morb Mortal Wkly Rep 2017; 66: 1369-1373

[2] Jensen MD, Ryan DH, Apovian CM et al. 2013 AHA/ACC/TOS guideline for the management of overweight and obesity in adults: a report of the American College of Cardiology/American Heart Association Task Force on Practice Guidelines and The Obesity Society. J Am Coll Cardiol 2014; 63: 2985-3023

[3] Blanco DG, Funes DR, Giambartolomei G et al. Laparoscopic sleeve gastrectomy versus Roux-en-Y gastric bypass in cardiovascular risk reduction: A match control study. Surg Obes Relat Dis 2019; 15: 14 20

[4] Shimada Y], Gibo K, Tsugawa Y et al. Bariatric surgery is associated with lower risk of acute care use for cardiovascular disease in obese adults. Cardiovasc Res 2019; 115: 800 - 806

[5] Chambers AP, Jessen L, Ryan KK et al. Weight-independent changes in blood glucose homeostasis after gastric bypass or vertical sleeve gastrectomy in rats. Gastroenterology 2011; 141: 950 - 958 
[6] Stefater MA, Sandoval DA, Chambers AP et al. Sleeve gastrectomy in rats improves postprandial lipid clearance by reducing intestinal triglyceride secretion. Gastroenterology 2011; 141: 939-949 e1-4

[7] Zechner JF, Mirshahi UL, Satapati S et al. Weight-independent effects of roux-en-Y gastric bypass on glucose homeostasis via melanocortin4 receptors in mice and humans. Gastroenterology 2013; 144: $580-$ 590 e7

[8] Stevens J, Oakkar EE, Cui Z et al. US adults recommended for weight reduction by 1998 and 2013 obesity guidelines, NHANES 2007-2012. Obesity 2015; 23: $527-531$

[9] Estimate of Bariatric Surgery Numbers, 2011-2017. 2018: https:// asmbs.org/resources/estimate-of-bariatric-surgery-numbers

[10] Acosta A, Abu Dayyeh BK, Port JD et al. Recent advances in clinical practice challenges and opportunities in the management of obesity. Gut 2014; 63: 687-695

[11] Ryan KK, Tremaroli V, Clemmensen C et al. FXR is a molecular target for the effects of vertical sleeve gastrectomy. Nature 2014; 509: $183-188$

[12] Oberbach A, Schlichting N, Heinrich M et al. Gastric mucosal devitalization reduces adiposity and improves lipid and glucose metabolism in obese rats. Gastrointest Endosc 2018; 87: 288-299 e6

[13] Kumbhari V, Lehmann S, Schlichting $N$ et al. Gastric mucosal devitalization is safe and effective in reducing body weight and visceral adiposity in a porcine model. Gastrointest Endosc 2018; 88: 175 - 184 e1

[14] Estimate of Bariatric Surgery Numbers,2011-2017. 2018: https:// asmbs.org/resources/estimate-of-bariatric-surgery-numbers

[15] Whelton PK, Carey RM, Aronow WS et al. 2017 ACC/AHA/AAPA/ABC/ ACPM/AGS/APhA/ASH/ASPC/NMA/PCNA Guideline for the Prevention, Detection, Evaluation, and Management of High Blood Pressure in Adults: Executive Summary: A Report of the American College of Cardiology/American Heart Association Task Force on Clinical Practice Guidelines. J Am Coll Cardiol 2018; 71: 2199-2269

[16] Sletten AC, Peterson LR, Schaffer JE. Manifestations and mechanisms of myocardial lipotoxicity in obesity. J Intern Med 2018; 284: 478 491

[17] Directive 2010/63/EU of the European Parliament and of the Council of 22 September 2010 on the protection of animals used for scientific purposes.https://eur-lex.europa.eu/legal-content/EN/TXT/?uri=celex \%3A32010L0063

[18] Oberbach A, Schlichting N, Heinrich M et al. Weight loss surgery improves the metabolic status in an obese rat model but does not affect bladder fibrosis associated with high fat diet feeding. Int J Obes (Lond) 2014; 38: 1061 - 1067

[19] Oberbach A, Jehmlich N, Schlichting $N$ et al. Molecular fingerprint of high fat diet induced urinary bladder metabolic dysfunction in a rat model. PLoS One 2013; 8: e66636

[20] Saydah S, Bullard KM, Cheng Y et al. Trends in cardiovascular disease risk factors by obesity level in adults in the United States, NHANES 1999-2010. Obesity (Silver Spring) 2014; 22: $1888-1895$

[21] Harsha DW, Bray GA. Weight loss and blood pressure control (Pro). Hypertension 2008; 51: $1420-1425$; discussion 5

[22] Hubert HB, Feinleib M, McNamara PM et al. Obesity as an independent risk factor for cardiovascular disease: a 26-year follow-up of participants in the Framingham Heart Study. Circulation 1983; 67: 968-977
[23] Huang Z, Willett WC, Manson JE et al. Body weight, weight change, and risk for hypertension in women. Ann Intern Med 1998; 128: 81 88

[24] Hall JE. The kidney, hypertension, and obesity. Hypertension 2003; 41: $625-633$

[25] Lim SS, Vos T, Flaxman AD et al. A comparative risk assessment of burden of disease and injury attributable to 67 risk factors and risk factor clusters in 21 regions, 1990-2010: a systematic analysis for the Global Burden of Disease Study 2010. Lancet 2012; 380: 2224-2260

[26] Lewington S, Clarke R, Qizilbash $N$ et al. Age-specific relevance of usual blood pressure to vascular mortality: a meta-analysis of individual data for one million adults in 61 prospective studies. Lancet 2002; 360: $1903-1913$

[27] Rapsomaniki E, Timmis A, George J et al. Blood pressure and incidence of twelve cardiovascular diseases: lifetime risks, healthy life-years lost, and age-specific associations in 1.25 million people. Lancet 2014; 383: 1899-1911

[28] Juonala M, Magnussen CG, Berenson GS et al. Childhood adiposity, adult adiposity, and cardiovascular risk factors. N Engl J Med 2011; 365: $1876-1885$

[29] Wang JG, Staessen JA, Franklin SS et al. Systolic and diastolic blood pressure lowering as determinants of cardiovascular outcome. Hypertension 2005; 45: $907-913$

[30] Turnbull F, Neal B, Algert C et al. Effects of different blood pressurelowering regimens on major cardiovascular events in individuals with and without diabetes mellitus: results of prospectively designed overviews of randomized trials. Arch Intern Med 2005; 165: 1410 1419

[31] Schmieder RE, Hilgers KF, Schlaich MP et al. Renin-angiotensin system and cardiovascular risk. Lancet 2007; 369: $1208-1219$

[32] Marcheselli S, Micieli G. Renin-angiotensin system and stroke. Neurol Sci 2008; 29: S277-S278

[33] Joseph J], Echouffo Tcheugui JB, Effoe VS et al. Renin-angiotensin-aldosterone system, glucose metabolism and incident Type 2 diabetes mellitus: MESA. J Am Heart Assoc 2018; 7: e009890

[34] Engeli S, Bohnke J, Gorzelniak K et al. Weight loss and the renin-angiotensin-aldosterone system. Hypertension 2005; 45: 356- 362

[35] Mao Y, Tokudome T, Kishimoto I. Ghrelin and blood pressure regulation. Curr Hypertens Rep 2016; 18: 15

[36] Goldberg IJ, Reue K, Abumrad NA et al. Deciphering the role of lipid droplets in cardiovascular disease: a report from the 2017 National Heart, Lung, and Blood Institute Workshop. Circulation 2018; 138: $305-315$

[37] Lang PD, Insull W Jr. Lipid droplets in atherosclerotic fatty streaks of human aorta. J Clin Invest 1970; 49: 1479-1488

[38] Itabe $\mathrm{H}$, Yamaguchi T, Nimura $\mathrm{S}$ et al. Perilipins: a diversity of intracellular lipid droplet proteins. Lipids Health Dis 2017; 16: 83

[39] Mazzali G, Fantin F, Zoico E et al. Heart fat infiltration in subjects with and without coronary artery disease. J Clin Endocrinol Metab 2015; 100: $3364-3371$

[40] Conte M, Franceschi C, Sandri M et al. Perilipin 2 and age-related metabolic diseases: a new perspective. Trends Endocrinol Metab 2016; 27: $893-903$

[41] Bosma M, Hesselink MK, Sparks LM et al. Perilipin 2 improves insulin sensitivity in skeletal muscle despite elevated intramuscular lipid levels. Diabetes 2012; 61: 2679-2690 